\title{
Characterization of Fabry Disease in 352 Pediatric Patients in the Fabry Registry
}

\author{
ROBERT J. HOPKIN, JOHN BISSLER, MARYAM BANIKAZEMI, LORNE CLARKE, CHRISTINE M. ENG, \\ DOMINIQUE P. GERMAIN, ROBERTA LEMAY, ANNA TYLKI-SZYMANSKA, AND WILLIAM R. WILCOX
}

\begin{abstract}
Division of Human Genetics [R.J.H.] and Division of Nephrology and Hypertension [J.B.], Cincinnati Children's Hospital Medical Center, Cincinnati, Ohio 45229; Department of Pediatrics [R.J.H., J.B.], University of Cincinnati College of Medicine, Cincinnati, Ohio 45229; Departments of Neurology and Pediatrics [M.B.], New York University, New York, New York 10016; Department of Medical Genetics [L.C.], University of British Columbia, Vancouver, British Columbia V6H 3N1, Canada; Department of Molecular and Human Genetics [C.M.E.], Baylor College of Medicine, Houston, Texas 77030; Hôpital Raymond Poincaré (AP-HP) [D.P.G.], University of Versailles, Saint Quentin en Yvelines, 92380 Garches, France; Biomedical Operations [R.L.], Genzyme Corporation, Cambridge, Massachusetts 02142; Department of Pediatrics [A.T.-S.], Klinika Chorob Metabolicznych, Warsaw 04-736, Poland; Medical Genetics Institute [W.R.W.], Cedars-Sinai Medical Center, Los Angeles, California 90048; Department of Pediatrics [W.R.W.], UCLA School of Medicine,
\end{abstract} Los Angeles, California 90095

\begin{abstract}
Fabry disease is an X-linked lysosomal disease caused by deficiency of $\alpha$-galactosidase A. Signs and symptoms of Fabry disease occurring during childhood and adolescence were characterized in 352 Fabry Registry patients. At enrollment (median age 12 year), $77 \%$ of males and $51 \%$ of females reported symptoms. The median age of symptom onset was 6 year in males and 9 year in females. The most frequent symptom, neuropathic pain, was reported by $59 \%$ of males (median age 7 year) and $41 \%$ of females (median age 9 year). Gastrointestinal symptoms were reported by $18 \%$ of children (median age 5 year in males and 9.5 year in females). Males exhibited height and weight values below the US 50th percentile. Females had weight values above the US 50th percentile. A few patients had serious renal and cardiac manifestations, stage 2 or 3 chronic kidney disease $(n=3)$, arrhythmia $(n=9)$, and left ventricular hypertrophy $(n=3)$. Thus, many pediatric Fabry patients report early symptoms, particularly pain, gastrointestinal symptoms, and impaired quality of life. Some children experience major complications during the pediatric years. (Pediatr Res 64: 550-555, 2008)
\end{abstract}

$\mathrm{F}$ abry disease is an X-linked disorder characterized by deficient activity of the lysosomal hydrolase, $\alpha$-galactosidase A (1). Affected patients cannot effectively metabolize membrane glycosphingolipids, particularly globotriaosylceramide (GL-3), resulting in its accumulation in the vascular endothelium of various tissues (2). Accumulation of GL-3 eventually leads to life-threatening renal, cardiac, and cerebrovascular complications, typically during the third to fifth decades of life $(2,3)$. Enzyme replacement therapy (ERT) reduces GL-3 accumulation in plasma and in the vascular endothelium of several tissues and attenuates many manifestations of the disease in adults (4-7). However, it is unclear whether initiation of ERT during childhood can prevent the end-organ manifestations of Fabry disease that occur during adulthood (8-10).

Received February 26, 2008; accepted June 2, 2008.

Correspondence: Robert J. Hopkin, M.D., Cincinnati Children's Hospital Medical Center, 3333 Burnet Avenue, Cincinnati, OH 45229-3039; e-mail: rob.hopkin@cchmc.org

W.R.W. was supported by a Winnick Family Clinical Development Scholar Award.
The symptoms of Fabry disease most often experienced by children include pain, gastrointestinal dysfunction, hypohidrosis, and abnormal heat and cold tolerance (11-13). Although not life-threatening, these symptoms clearly impact the health and function of affected children. A better understanding of the manifestations of Fabry disease during childhood will provide valuable information about how this disorder progresses.

To date, 3 descriptive studies have been published on Fabry disease in a total of 142 pediatric patients (11-13). Three clinical studies evaluated the effects of ERT in 53 children (8-10). The Fabry Registry, an observational database that tracks the natural history and outcomes of patients with Fabry disease, has now collected detailed clinical data from 352 pediatric patients. These data were analyzed to better understand Fabry disease during childhood and adolescence, before treatment with ERT.

\section{METHODS}

Fabry Registry. The Fabry Registry is administered by Genzyme Corporation as a postmarketing regulatory commitment. All patients with Fabry disease, whether or not they receive ERT and irrespective of the commercial product with which they are treated, are eligible to enroll. Participation in the Fabry Registry is voluntary. The parents or legal guardians of pediatric patients (age $<18$ year) provided informed consent, and consent could be withdrawn at any time. At each site, patient participation was approved and monitored by local institutional review boards or by a governing body such as the Western Institutional Review Board, for smaller sites that did not have a local institutional review board. Treating physicians determine the frequency of necessary assessments according to a patient's individualized need for medical care and routine follow-up. A recommended schedule of assessments for patients with Fabry disease is available at http://www.fabryregistry.com.

Data analysis and statistics. Data included in these analyses were obtained before initiation of ERT or from patients who had never received ERT, except where indicated otherwise. Data were collected through standardized case report forms, which are submitted to the Fabry Registry for central analysis. The following statistical analyses were used: two-sample median test to compare age at symptom onset by gender; two-sample $t$ test with equal variances to compare age at onset of symptoms and to compare "average

\footnotetext{
Abbreviations: BPI, Brief Pain Inventory; eGFR, estimated glomerular filtration rate; ERT, enzyme replacement therapy; GL-3, globotriaosylceramide; SF-36 ${ }^{\circledR}$, Short Form 36 Health Status Questionnaire
} 
pain" scores in the Brief Pain Inventory (BPI) instrument between genders; two-sample $t$ test with unequal variances to compare "worst pain" BPI scores between genders; two sample $t$ test to compare Short Form 36 (SF-36 ${ }^{\circledR}$ ) Health Status Questionnaire scores in Fabry patients to scores of the general population; and Fisher's exact test to compare percentage of males and females presenting with neuropathic pain, gastrointestinal symptoms, abnormal sweating, heat intolerance, cold intolerance, chronic pain, pain therapy, and acute pain crises.

Cardiovascular assessments. Cardiovascular examinations included electrocardiograms and/or echocardiograms. Treating physicians indicated whether any cardiac abnormalities were present, including sinus rhythm abnormalities, conduction abnormalities, valvular dysfunction, wall motion abnormalities, hypertrophy, and left ventricular hypertrophy.

Renal assessments. Renal function was assessed by calculation of estimated GFR (eGFR), using the Schwartz formula:

$$
\operatorname{eGFR}\left(\frac{\frac{\mathrm{mL}}{\min }}{1.73 \mathrm{~m}^{2}}\right)=\frac{k \times H t(\mathrm{~cm})}{\text { serumCr }\left(\frac{\mathrm{mg}}{\mathrm{dL}}\right)}
$$

where the constant $k$ is set at 0.55 in children and adolescent girls, and 0.70 in adolescent boys (14). Serum creatinine values $<0.2 \mathrm{mg} / \mathrm{dL}$ were excluded from the analyses. Height measurements obtained \pm 3 mo from the time the corresponding serum creatinine values were obtained were used to calculate eGFR.

Definition of cardiac, renal, and cerebrovascular events. Cardiovascular events were defined as myocardial infarction, significant cardiac procedures (such as pacemaker placement, bypass, stent placement, valve replacement, transplantation, etc.), arrhythmia, angina pectoris, congestive heart failure, or left ventricular hypertrophy. Cerebrovascular clinical events were defined as stroke. Renal clinical events were defined as dialysis or kidney transplantation.

Pain assessments. Pain was measured in patients age $\geq 12$ to $<18$ year with the BPI instrument, which assesses pain by asking patients to evaluate pain on a scale of 0 to 10 , where 0 is no pain and 10 is pain "as bad as you can imagine." BPI scores were divided into severity categories with $0-3$ representing mild, 4-6 moderate, and 7-10 severe pain.

Quality of life. Health-related quality of life was measured in patients age $\geq 14$ to $<18$ year with the SF- $36^{\circledR}$ health-related quality of life survey (15). Scores for the 8 subscales (Physical Functioning, Role Physical, Body Pain, General Health, Vitality, Social Functioning, Role Emotional, and Mental Health) are based on a 100-point scale, with a higher score indicating better quality of life. This instrument is suitable for use in patients' age 14 and older (16). However, the youngest age category for which gender-specific SF-36 ${ }^{\circledR}$ norm data are available from the general population is 18 to $<25$ year (personal communication, Michael DeRosa, QualityMetric, Lincoln, RI). Therefore, gender-specific SF-36 ${ }^{\circledR}$ scores of patients age $\geq 14$ to $<18$ year in the Fabry Registry were compared with gender-specific United States population norms from the $\geq 18$ to $<25$ age group as the closest available control (15).

\section{RESULTS}

Patient demographics and onset of symptoms. A total of 352 pediatric patients (age $<18$ year at the time of enrollment), including 194 males (55.1\%) and 158 females (48.9\%) were identified as of November 2, 2007. Age at enrollment ranged from 0 to 17 year with a median age of 12 year for both genders. At enrollment, $76.8 \%$ of males and $50.6 \%$ of females reported symptoms. Demographic data are summarized in Table 1. The median age at diagnosis was 9 year, for both males and females, with 35 males $(18.0 \%)$ and 20 females (12.6\%) diagnosed before the onset of symptoms. The relatively young age at diagnosis reflects the fact that most patients had family members previously diagnosed with Fabry disease.

Males generally experienced the onset of symptoms at an earlier age than females, at a median age of 6 year, compared with 9 year in females $(p<0.01)$. Figure 1 summarizes presenting symptoms reported by children at the time of enrollment. The symptom most frequently reported was neuropathic pain, which included episodic pain crises, chronic
Table 1. Demographics and disease characteristics of pediatric Fabry Registry patients

\begin{tabular}{|c|c|c|}
\hline Parameter & $\begin{array}{l}\text { Male pediatric } \\
\text { patients }\end{array}$ & $\begin{array}{c}\text { Female pediatric } \\
\text { patients }\end{array}$ \\
\hline \multicolumn{3}{|c|}{$\begin{array}{l}\text { Total number of pediatric patients in } \\
\text { the Fabry Registry, } \\
\text { (age }<18 \text { at enrollment) }\end{array}$} \\
\hline $\mathrm{n}$ & 194 & 158 \\
\hline \multicolumn{3}{|c|}{ Age in yrs at registry enrollment } \\
\hline Mean (SD) & $11.4(4.52)$ & $11.1(4.43)$ \\
\hline Median (range) & $12(<1 *, 17)$ & $12(<1 *, 17)$ \\
\hline \multicolumn{3}{|l|}{ Ethnicity, n (\%) } \\
\hline Caucasian & $124(63.9)$ & $109(69.0)$ \\
\hline Black & $8(4.1)$ & $2(1.3)$ \\
\hline Hispanic & $18(9.3)$ & $17(10.8)$ \\
\hline Asian & $14(7.2)$ & 0 \\
\hline Other & $11(5.7)$ & $5(3.2)$ \\
\hline Not stated & $19(9.8)$ & $25(15.9)$ \\
\hline \multicolumn{3}{|c|}{$\begin{array}{l}\text { Family members diagnosed } \\
\text { with Fabry, } \mathrm{n}(\%)\end{array}$} \\
\hline Yes & $156(80.4)$ & $131(82.9)$ \\
\hline No & $12(6.2)$ & $1(0.6)$ \\
\hline Not stated & $26(13.4)$ & $26(16.5)$ \\
\hline \multicolumn{3}{|c|}{ Age in yrs at first Fabry symptoms } \\
\hline $\mathrm{N}$ & 132 & 65 \\
\hline Mean (SD) & $6.6(3.27)$ & $8.4(3.94)$ \\
\hline Median (range) & $6\left(<1^{*}, 14\right)$ & $9\left(<1^{*}, 16\right)$ \\
\hline \multicolumn{3}{|l|}{ Age category, $\mathrm{n}(\%)$} \\
\hline $0-<5$ yrs & $37(19.1)$ & $12(7.6)$ \\
\hline$\geq 5-<8$ yrs & $41(21.1)$ & $12(7.6)$ \\
\hline$\geq 8-<12$ yrs & $41(21.1)$ & $28(17.7)$ \\
\hline$\geq 12-<15$ yrs & $13(6.7)$ & $8(5.1)$ \\
\hline$\geq 15-<18$ yrs & 0 & $5(3.2)$ \\
\hline Not stated & $62(32.0)$ & $93(58.9)$ \\
\hline \multicolumn{3}{|c|}{ Age in yrs at Fabry diagnosis } \\
\hline $\mathrm{n}$ & 178 & 153 \\
\hline Mean (SD) & $8.6(4.75)$ & $8.3(4.88)$ \\
\hline Median (range) & $9(<1 *, 17)$ & $9(<1 *, 17)$ \\
\hline
\end{tabular}

* Note that patient age was expressed as the integer portion of the patient's calculated age, as of November 2007. Thus, patients less than $1 \mathrm{yr}$ of age are designated as age $<1$.

pain, and acroparesthesias. Neuropathic pain was reported as a presenting symptom more frequently by males $(58.8 \%)$ than by females $(40.5 \%, p<0.001)$. Males also reported an earlier onset of neuropathic pain, with a median age of onset at 7 year, compared with 9 year in females $(p<0.05)$.

Gastrointestinal symptoms captured by the Fabry Registry include abdominal pain and diarrhea. At enrollment, $17.9 \%$ of patients reported having gastrointestinal symptoms, which were a presenting symptom more frequently $(p<0.005)$ and at an earlier age $(p<0.02)$ among males $(23.2 \%$ at a median age of onset of 5 year) than among females $(11.4 \%$ at a median age of onset of 9.5 year). However, when data from clinical follow-up assessments were combined with enrollment medical history data, similar percentages of males and females reported abdominal pain (26.7\% of all patients) and diarrhea (19.3\% of all patients). Overall, $14.2 \%$ of pediatric patients presented with abnormal skin findings (i.e., angiokeratomas), at a median age of onset of 7 year in males and 9.5 year in females $(p=0.29$, NS). Angiokeratomas were also reported as a presenting abnormality more frequently among males $(19.6 \%)$ than females $(7.6 \%)(p<0.001)$. 


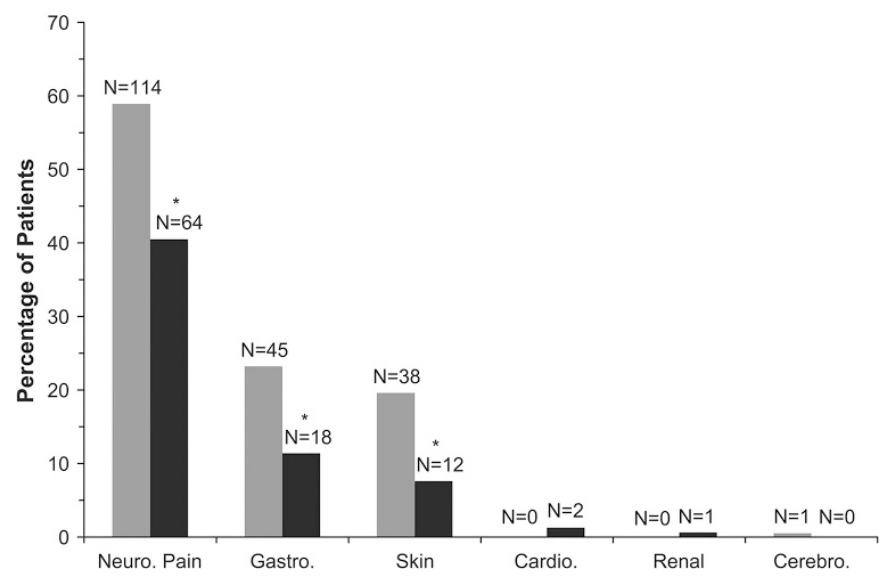

Figure 1. Presenting Fabry signs and symptoms in major organ systems among pediatric patients at enrollment in the Fabry Registry. Males: gray bars; females: black bars. Neuro Pain $=$ neuropathic pain, Gastro $=$ gastrointestinal, Cardio $=$ cardiovascular, Cerebro $=$ cerebrovascular. Data are expressed as the percentage of male $(n=194)$ and female $(n=158)$ patients reporting signs or symptoms within key organ systems at enrollment. The number of patients reporting signs or symptoms within each category is indicated above each bar. ${ }^{*} p<0.05$ by Fisher's exact test (indicates difference between percentages of males and females reporting these symptoms).
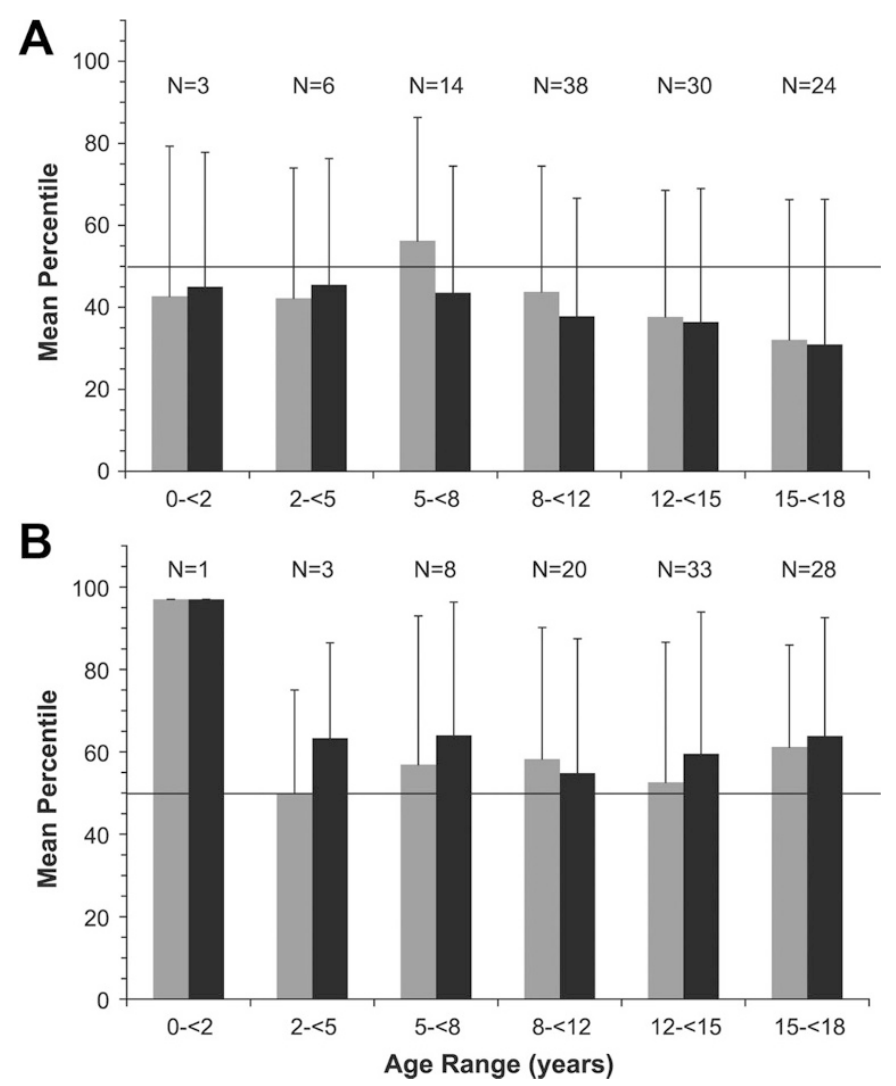

Figure 2. Mean height and weight percentiles in male (A) and female (B) pediatric Fabry Registry patients. Height: gray bars; weight: black bars. The horizontal line on each graph indicates the 50th percentile. Error bars indicate SD. Patients' earliest available data were included within each age category. Height and weight percentiles are based on gender-specific growth charts from the Center for Disease Control (CDC) and National Center for Health Statistics (NCHS). The number of patients reporting height and weight data within each age category is indicated above the bars. Note that the CDC/ NCHS charts are scaled for a US population, while the patients in the Fabry Registry represent an international cohort.
Anthropomorphic data. Height and weight measurements were stratified across 6 age categories, as shown in Figure 2. Among males, median height and weight percentiles were below the US 50th percentile (median 25th percentile, $p<0.001$ for both height and weight). Six males exhibited height percentiles below the normal range (i.e., below the 3 rd percentile) and 7 males exhibited weight percentiles below the normal range in at least 1 age group. Conversely, the median weight percentile for females was significantly greater than the US 50th percentile (median weight 75 th percentile, $p<0.001$ ). Six females exhibited weight percentiles above the normal range (i.e., above the 97th percentile) in at least 1 age group. The median height percentile among females was not different from the US 50th percentile (median 50th percentile, mean 57.2\%). Males exhibited more variability in height and weight (i.e., a greater range of percentile values), compared with females. Among males, mean height and weight percentiles tended to decline with age.

Sweating and heat and cold tolerance. When data from clinical follow-up assessments were combined with enrollment medical history data, abnormal sweating was reported by $28.4 \%$ of males versus $22.2 \%$ of females ( $p=0.22$, NS); abnormal heat tolerance was reported by $28.9 \%$ of males versus $24.7 \%$ of females $(p=0.40, \mathrm{NS})$. Abnormal cold intolerance was reported by $18.0 \%$ of males versus $10.1 \%$ of females $(p<0.05)$.

Serious disease manifestations-Vital organ function. At enrollment, a small number of patients presented with abnormal findings that were related to vital organ function. One patient presented with unspecified cerebrovascular signs, 1 patient presented with renal signs, and 2 patients presented with cardiovascular signs, as shown in Figure 1.

Although only 2 patients presented with cardiovascular signs at the time of enrollment, additional cardiac abnormalities were detected in some pediatric patients at subsequent follow-up clinical assessments. The most common cardiac abnormality detected was valvular dysfunction, which was found in 21 males (22.6\%) and 11 females (13.9\%), based on the 93 males and 79 females for whom cardiac examination data were available. Nine patients experienced arrhythmias during the pediatric natural history period including 7 males (median age 16, range 12 to 19 year) and 2 females (both at age 12). Five of the males were reported to have sinus bradycardia and 2 were reported to have ectopic atrial rhythms; both females had sinus bradycardia. Each of these patients had been diagnosed with Fabry disease before experiencing an arrhythmia. Several patients also exhibited conduction abnormalities and/or left ventricular hypertrophy, as indicated in Figure 3.

The occurrence of major clinical events (cardiovascular, renal, or cerebrovascular events, as defined in Methods) was also analyzed, regardless of whether these events occurred before or after enrollment and irrespective of treatment status. When these data were considered, 1 additional patient had an arrhythmia approximately 1 year after the initiation of ERT, a male at age 18. Another male patient was reported to have undergone an unspecified significant cardiac procedure at the age of 14 year, which preceded his diagnosis by 2 year. No patients experienced major cerebrovascular or renal clinical 


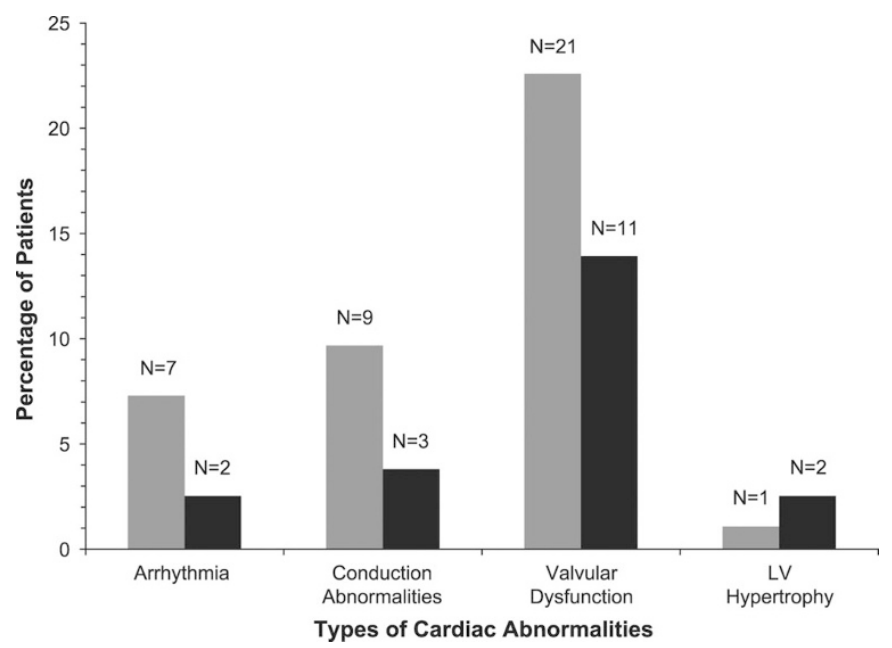

Figure 3. Percentage of pediatric Fabry Registry patients ever exhibiting cardiac abnormalities. Males: gray bars; females: black bars. For arrhythmias, percentages were calculated based on the number of patients with echocardiographic or electrocardiographic data available plus any patients who reported arrhythmia as a cardiac clinical event but who did not report cardiac examination data (96 males and 79 females). All other percentages were calculated based on the number of patients for whom cardiac examination data were available (93 males and 79 females). The number of patients in each group is indicated above each bar.

Table 2. Summary of renal assessments among pediatric Fabry Registry patients

\begin{tabular}{clccc}
\hline $\begin{array}{c}\text { Laboratory } \\
\text { parameter }\end{array}$ & Statistic & $\begin{array}{c}\text { Males } \\
\mathrm{N}=194\end{array}$ & $\begin{array}{c}\text { Females } \\
\mathrm{N}=158\end{array}$ & $\begin{array}{c}\text { All patients } \\
\mathrm{N}=352\end{array}$ \\
\hline $\begin{array}{c}\text { Serum creatinine } \\
(\mathrm{mg} / \mathrm{dL})\end{array}$ & $\mathrm{n}$ & 100 & 94 & 194 \\
& Mean (SD) & $0.6(0.25)$ & $0.6(0.16)$ & $0.6(0.21)$ \\
& Median & 0.6 & 0.6 & 0.6 \\
Estimated GFR & Min, Max & $0.3,2.4$ & $0.3,1.2$ & $0.3,2.4$ \\
$\left(\mathrm{~mL} / \mathrm{min} / 1.73 \mathrm{~m}^{2}\right)$ & Mean (SD) & $150(36.8)$ & $144(30.9)$ & $147(33.9)$ \\
& Median & 146 & 141 & 143 \\
Total urinary & Min, Max & 56,269 & 73,227 & 56,269 \\
protein & $\mathrm{n}$ & 42 & 44 & 86 \\
$(\mathrm{mg} / 24$ hr) & Mean (SD) & $114(166.9)$ & $118(127.9)$ & $116(147.3)$ \\
& Median & 94 & 91 & 91 \\
\hline
\end{tabular}

Data shown represent natural history data. If patients received ERT, only data occurring before first ERT are included. GFR was estimated by the Schwartz formula, using heights \pm 3 months from the serum creatinine value. Serum creatinine values ranging from 0.2 to $2.4 \mathrm{mg} / \mathrm{dL}$ were included in these analyses. For 24-hr protein levels, any values less than $10 \mathrm{mg}$ were set to a minimum cutoff of $10 \mathrm{mg}$; no patients exhibited 24-hr protein values greater than $1134 \mathrm{mg} / 24 \mathrm{hr}$.

events (defined as having a stroke, renal transplant, or chronic dialysis).

In terms of renal function, eGFR values were $>140 \mathrm{~mL} /$ min/1.73 $\mathrm{m}^{2}$ among both genders (Table 2), and 32 patients $(22.2 \%)$ exhibited eGFR $>170 \mathrm{~mL} / \mathrm{min} / 1.73 \mathrm{~m}^{2}$. We considered the possibility that these high eGFR values could be related to patients' muscle mass and therefore examined their height and body size. Linear regression analyses indicated that there were no correlations between eGFR and height, eGFR and weight, or eGFR and body mass index, in either males or females (all correlation coefficients $<0.27$ ), suggesting that growth disturbance cannot explain these high eGFR values.
Three patients exhibited abnormally low eGFR values (defined as $<90 \mathrm{~mL} / \mathrm{min} / 1.73 \mathrm{~m}^{2}$ ); a 7-year-old male with eGFR of $89 \mathrm{~mL} / \mathrm{min} / 1.73 \mathrm{~m}^{2}$, a 4-year-old male with eGFR of 56 $\mathrm{mL} / \mathrm{min} / 1.73 \mathrm{~m}^{2}$, and a 13 -year-old female with eGFR of 73 $\mathrm{mL} / \mathrm{min} / 1.73 \mathrm{~mm}^{2}$. Among the 86 patients with $24-\mathrm{h}$ urinary protein data available, 4 males and 5 females ranging in age from 2 to 17 year exhibited proteinuria (defined as total protein $>150 \mathrm{mg} / 24 \mathrm{~h}$ ); the highest proteinuria value measured in any patient was $1134 \mathrm{mg} / 24 \mathrm{~h}$ in a 15 -year-old male (Table 2). Four additional patients (3 males and 1 female) exhibited microalbuminuria (defined as $>30 \mathrm{mg} / 24 \mathrm{~h}$ ), among the 46 patients for whom data were available. Among the 10 patients with proteinuria or microalbuminuria for whom eGFR data were available, 7 had eGFR $>140$. The mean eGFR level among these few patients $(163 \pm 39.7, n=10)$ was not significantly different $(p=0.17)$ than mean eGFR level among the rest of the patients in the cohort $(145.8 \pm 33.34, n=134)$.

Quality of life and pain. SF-36 ${ }^{\circledR}$ questionnaire data were available from 10 male patients and 26 female patients $\geq 14$ year of age. Fabry Registry males ages $\geq 14$ to $<18$ year reported significantly poorer quality of life than males age $\geq 18$ to $<25$ in the general US population in 7 of the $8 \mathrm{SF}-36^{\circledR}$ subscales (all but Role Emotional). Fabry Registry females ages $\geq 14$ to $<18$ year reported significantly poorer quality of life in 2 of the 8 subscales (Body Pain and General Health), compared with females age $\geq 18$ to $<25$ in the general US population.

Sixty-six male patients reported ever experiencing chronic pain $(34.0 \%)$, as did 51 females $(32.3 \%)$ [ $p=0.73$, NS]. Fewer patients $(8.0 \%)$ reported ever experiencing acute pain crises, including 19 males $(9.8 \%)$ and 9 females $(5.7 \%)$

Table 3. BPI scores among pediatric patients $\geq 12$ yrs of age in the Fabry Registry

\begin{tabular}{cccc}
\hline \multicolumn{1}{c}{ Parameter } & Males & Females & $\begin{array}{c}\text { All } \\
\text { patients }\end{array}$ \\
\hline $\begin{array}{c}\text { Total number of patients } \geq 12 \text { to } \\
<18 \text { yrs of age with BPI data } \dagger\end{array}$ & 19 & 26 & 45 \\
First recorded BPI score for worst & & & \\
$\quad$ pain in the past 24 hrs & & & \\
Mean (SD) & $4.4(3.51)$ & $1.5(2.45) *$ & $2.7(3.23)$ \\
Median (range) & $5(0,10)$ & $0(0,9)$ & $1(0,10)$ \\
0-3 (mild) & $9(47.4 \%)$ & $22(84.6 \%)$ & $31(68.9 \%)$ \\
4-7 (moderate) & $4(21.1 \%)$ & $3(11.5 \%)$ & $7(15.6 \%)$ \\
$8-10$ (severe) & $6(31.6 \%)$ & $1(3.8 \%)$ & $7(15.6 \%)$ \\
First recorded BPI score & & & \\
$\quad$ for average pain§ & & & \\
Mean (SD) & $3.8(2.54)$ & $2.3(2.47) \mathscr{1}$ & $3.0(2.59)$ \\
Median (range) & $3(0,10)$ & $1.5(0,8)$ & $3(0,10)$ \\
$0-3$ (mild) & $10(52.6 \%)$ & $18(69.2 \%)$ & $28(62.2 \%)$ \\
$4-7$ (moderate) & $7(36.8 \%)$ & $7(26.9 \%)$ & $14(31.1 \%)$ \\
$8-10$ (severe) & $2(10.5 \%)$ & $1(3.8 \%)$ & $3(6.7 \%)$ \\
\hline
\end{tabular}

Data shown represent natural history data. If patients received ERT, only data occurring before first ERT are included.

$* p<0.02$ by $t$-test (difference in mean scores between males and females).

$\dagger$ The BPI has been validated for patients $\geq 12$ yrs of age. First available scores are summarized.

$\ddagger$ BPI Question 3: “Worst pain in the last $24 \mathrm{hr}$ " scored as 0 (no pain) to a high of 10 (pain as bad as you can imagine).

$\S$ BPI Question 5: "Average Pain" scored as 0 (no pain) to a high of 10 (pain as bad as you can imagine).

II Indicates not significant by $t$-test (no difference in mean scores between males and females, $p=0.25$ ). 
females $(p=0.17$, NS). Nineteen percent of all pediatric patients had ever been on pain therapy $(18.0 \%$ of males and $19.6 \%$ of females; $p=0.78$, NS).

BPI data were available from 45 patients ages $\geq 12$ to $<18$ year old (Table 3 ). Thirty-two percent of males reported experiencing their "worst pain in the past $24 \mathrm{~h}$ " as being "severe," compared with $3.4 \%$ of females. Mean BPI scores for this parameter were significantly higher in males than in females $(p<$ 0.02 ). Male patients also tended to report more severe "average pain" than female patients, but the difference between genders in mean BPI scores for average pain was not statistically significant $(p=0.25, \mathrm{NS})$.

Treatment with ERT in pediatric patients. Among the 352 pediatric patients in the Fabry Registry, 120 males (61.9\%) and 21 females (13.3\%) have ever received ERT. Patients were classified as symptomatic if they reported having Fabry symptoms at the time of enrollment. Among patients for whom such data were available, 69 of 149 symptomatic males $(46.3 \%)$ were on ERT at the time of enrollment, compared with only 10 of 80 symptomatic females (12.5\%). Two asymptomatic males, but no asymptomatic females were being treated with ERT at the time of enrollment. Among patients on ERT at the time of enrollment, the median age at initiation of ERT was 13 year in males and 11.5 year in females.

\section{DISCUSSION}

This study provides detailed analyses on a large, international cohort of pediatric patients with Fabry disease. All data reported are preERT, unless otherwise specified. Thus, these analyses represent the unmodified course of Fabry disease in an untreated population. These patients were diagnosed at a relatively early age (median 9 year), as the majority of patients had family members previously diagnosed with Fabry disease. There are certain limitations associated with this type of study (i.e., voluntary, observational data collection). As different patients may have had clinical assessments at various ages and time intervals, statistical analyses of these data must be interpreted with caution. In addition, while physicians are encouraged to enroll all Fabry patients in the registry regardless of overt signs and symptoms, individuals with more prominent signs and symptoms (typically males) may be more likely to enroll in the registry. These and other factors must be considered when interpreting these data.

The symptom most frequently experienced by this cohort was neuropathic pain, reported by $59 \%$ of males and $41 \%$ of females at a median age of 7 and 9 year, respectively. BPI scores indicated that $53 \%$ of males and $15 \%$ of females had experienced moderate or severe pain during the previous $24 \mathrm{~h}$. Despite this, most patients had not received any treatment for pain. This underscores the importance of better pain management in children with Fabry disease, regardless of other associated signs or symptoms.

Gastrointestinal symptoms were the second most common problem, reported by $27 \%$ of participants (including $19 \%$ with diarrhea), median age of onset of 5 year in males and 9.5 year in females. This is consistent with Ramaswami et al. (11), who also reported that abdominal cramps and diarrhea are the second most common presenting symptoms of Fabry disease. Although severity of these symptoms was not measured in this study, gastrointestinal disturbances can be severe enough to cause school absences and, in some cases, to mimic inflammatory bowel disease (17). Future studies of gastrointestinal symptoms in this population should include validated measures that can be compared with general population since gastrointestinal complaints are common in the general population.

Clearly, Fabry disease not only threatens long-term health, but it also poses a significant burden during childhood for both males and females. Future studies using tools designed for pediatric patients are needed to fully assess the burden of Fabry disease in this population.

Interestingly, males had median height and weight values below the US 50th percentile, particularly during adolescence, whereas females had median weight values above the US 50th percentile, but had normal height. This gender difference may be because Fabry disease is typically more severe in males than in females $(18,19)$. Alternatively, it may reflect distinct manifestations of the disease between genders.

Two of the 144 patients for whom eGFR data were available exhibited stage 2 chronic kidney disease and 1 patient exhibited stage 3 chronic kidney disease, indicating that some Fabry patients experience serious renal dysfunction during childhood. Most children exhibited eGFR values considerably higher than expected for healthy children and adolescents, which has been estimated as 110 to $140 \mathrm{mg} / \mathrm{mL} / \mathrm{min} / 1.73 \mathrm{~m}^{2}$ (20). The relatively high eGFR levels exhibited by these patients do not seem to be explained by the growth disturbance that was also seen in this population, although muscle mass was not measured directly. However, these data must be interpreted cautiously, as the predictive equations used to estimate GFR can overestimate GFR in patients who do not have chronic kidney disease $(20,21)$. Clearly, determination of actual GFR (i.e., by inulin or Tc99m diethylene triamine pentaacetate clearance) would provide a better measure of renal function. Nevertheless, it is possible that the elevated eGFR levels in these patients reflect renal hyperfiltration. Interestingly, 4 other studies of pediatric patients with Fabry disease have reported similar findings (i.e., relatively high eGFR levels in children) $(8-10,12)$. Nine patients exhibited proteinuria and 4 patients exhibited microalbuminuria, indicating some level of renal dysfunction. As GFR decline is likely to be a later manifestation of glomerular damage, we recommend obtaining timed urine protein values or spot protein:creatinine ratios to stage and monitor renal status among pediatric Fabry patients.

Only 2 of 352 patients exhibited overt cardiovascular signs at the time of enrollment. However, when findings from clinical follow-up assessments were included, a surprising number of serious cardiac abnormalities were identified among both genders. Three children had left ventricular hypertrophy ( 1 male and 2 females). Nine children experienced arrhythmias during the natural history period, including 7 males and 2 females, as early as age 12 . When all pediatric data were evaluated (regardless of ERT status), a total of 10 patients (8 males and 2 females) experienced arrhythmias. Early diagnosis and regular cardiac examinations, including 
electrocardiograms and echocardiography, are clearly an essential part of monitoring and care of pediatric patients with Fabry disease.

None of the children experienced cerebrovascular or renal events during the natural history period (defined as having a stroke, renal transplant, or undergoing chronic dialysis).

Not unexpectedly, males tended to experience all symptoms at an earlier age and at a higher prevalence than females. Originally, females were thought to experience only mild symptoms of Fabry disease (2). However, it has recently become clear that many females exhibit major complications of Fabry disease $(18,19)$. These data demonstrate that females also experience considerable symptoms and some serious manifestations of Fabry disease during childhood.

The prevalence of significant clinical events in the overall Fabry Registry population is much higher than what was observed in the pediatric population, with major renal, cardiovascular, and cerebrovascular events typically occurring during the fourth and fifth decades of life $(3,18)$. This age gap provides a potential opportunity to intervene and change the natural history of the disease for patients diagnosed during childhood. Ideally, treatment would alleviate the early symptoms as well as reduce or eliminate the risk of developing life-threatening complications later in life. Among adults, ERT has been shown to reduce glycolipid accumulation in plasma and tissue and to lessen various symptoms of the disease (4-7). Furthermore, ERT has been shown to be most effective when administered before prolonged accumulation of GL-3 can irreversibly damage renal, cardiovascular, and cerebrovascular tissues $(6,22)$. The pediatric studies published to date have not included enough patients with sufficient longitudinal data to determine whether initiation of ERT during childhood will prevent the major organ complications that occur in adulthood (8-10). Nevertheless, it is disconcerting that the majority of symptomatic children (53\% of males and $87 \%$ of females) had not been treated with ERT. Both prospective treatment trials and long-term follow-up studies are essential to assess the impact of treatment on this important group of patients.

In summary, children clearly experience substantial symptoms of Fabry disease. Pain, gastrointestinal symptoms, and impaired quality of life represent the immediate burden. We show for the first time, evidence of impaired physical growth among males with Fabry disease. Furthermore, a small percentage of children with Fabry disease experience serious cardiac and renal manifestations during childhood, emphasizing the importance of early diagnosis, careful monitoring, and appropriate intervention to prevent future complications.

Acknowledgments. The authors thank Michael DeRosa, MA (QualityMetric, Lincoln, RI) for assistance in analyzing the SF-36 ${ }^{\circledR}$ data. The authors also thank Dana BeitnerJohnson, Ph.D., Fanny O'Brien, Ph.D., and Marta Cizmarik, MS (Genzyme Corporation, Cambridge, MA) for assistance with manuscript writing and statistical analyses. The authors gratefully acknowledge the participation of 216 physicians in 38 countries around the world who contribute data to the Fabry Registry, their clinic staff, and especially the patients and their families.

\section{REFERENCES}

1. Brady RO, Gal AE, Bradley RM, Martensson E, Warshaw AL, Laster L 1967 Enzymatic defect in Fabry's disease: ceramide trihexosidase deficiency. N Engl J Med 276:1163-1167

2. Desnick RJ, Ioannou YA, Eng CM $2001 \alpha$-Galactosidase A deficiency: Fabry disease. In: Scriver CR, Beaudet AL, Sly WS, Valle D (eds) The Metabolic and Molecular Bases of Inherited Disease, 8th ed. McGraw Hill, New York, pp 3733 3774

3. Eng CM, Fletcher J, Wilcox WR, Waldek S, Scott CR, Sillence DO, Breunig F, Charrow J, Germain DP, Nicholls K, Banikazemi M 2007 Fabry disease: baseline medical characteristics of a cohort of 1765 males and females in the Fabry Registry. J Inherit Metab Dis 30:184-192

4. Eng CM, Banikazemi M, Gordon RE, Goldman M, Phelps R, Kim L, Gass A, Winston J, Dikman S, Fallon JT, Brodie S, Stacy CB, Mehta D, Parsons R, Norton K, O'Callaghan M, Desnick RJ 2001 A phase 1/2 clinical trial of enzyme replacement in Fabry disease: pharmacokinetic, substrate clearance, and safety studies. Am J Hum Genet 68:711-722

5. Schiffmann R, Kopp JB, Austin HA III, Sabnis S, Moore DF, Weibel T, Balow JE Brady RO 2001 Enzyme replacement therapy in Fabry disease: a randomized controlled trial. JAMA 285:2743-2749

6. Germain DP, Waldek S, Banikazemi M, Bushinsky DA, Charrow J, Desnick RJ, Lee P, Loew T, Vedder AC, Abichandani R, Wilcox WR, Guffon N 2007 Sustained, long-term renal stabilization after 54 months of agalsidase beta therapy in patients with Fabry disease. J Am Soc Nephrol 18:1547-1557

7. Wilcox WR, Banikazemi M, Guffon N, Waldek S, Lee P, Linthorst GE, Desnick RJ Germain DP; International Fabry Disease Study Group 2004 Long-term safety and efficacy of enzyme replacement therapy for Fabry disease. Am J Hum Genet 75:65-74

8. Ries M, Clarke JT, Whybra C, Timmons M, Robinson C, Schlaggar BL, Pastores G, Lien YH, Kampmann C, Brady RO, Beck M, Schiffmann R 2006 Enzyme replacement therapy with agalsidase alfa in children with Fabry disease. Pediatrics 118:924-932

9. Ramaswami U, Wendt S, Pintos-Morell G, Parini R, Whybra C, Leon Leal JA Santus F, Beck M 2007 Enzyme replacement therapy with agalsidase alfa in children with Fabry disease. Acta Paediatr 96:122-127

10. Wraith JE, Tylki-Szymanska A, Guffon N, Lien YH, Tsimaratos M, Vellodi A, Germain DP 2008 Safety and efficacy of enzyme replacement therapy with agalsidase beta: an international, open-label study in pediatric patients with Fabry disease. J Pediatr 152:563-570

11. Ramaswami U, Wendt S, Pintos-Morell G, Parini R, Whybra C, Leon Leal JA, Santus F, Beck M 2006 Clinical manifestations of Fabry disease in children: data from the Fabry Outcome Survey. Acta Paediatr 95:86-92

12. Ries M, Ramaswami U, Parini R, Lindblad B, Whybra C, Willers I, Gal A, Beck M 2003 The early clinical phenotype of Fabry disease: a study on 35 European children and adolescents. Eur J Pediatr 162:767-772

13. Ries M, Gupta S, Moore DF, Sachdev V, Quirk JM, Murray GJ, Rosing DR, Robinson C, Schaefer E, Gal A, Dambrosia JM, Garman SC, Brady RO, Schiffmann R 2005 Pediatric Fabry disease. Pediatrics 115:e344-e355

14. Schwartz GJ, Brion LP, Spitzer A 1987 The use of plasma creatinine concentration for estimating glomerular filtration rate in infants, children and adolescents. Pediatr Clin North Am 34:571-590

15. Ware JE, Snow KK, Kosinski M, Gandek B 1993 SF- $36^{\circledR}$ Health Survey Manual and Interpretation Guide. New England Medical Center, The Health Institute, Boston, MA

16. Gandek B, Ware JE 1998 Methods for validating and norming translations of health status questionnaires: the IQOLA Project approach. J Clin Epidemiol 51:953-959

17. Hoffmann B, Schwarz M, Mehta A, Keshav S; Fabry Outcome Survey European Investigators 2007 Gastrointestinal symptoms in 342 patients with Fabry disease: prevalence and response to enzyme replacement therapy. Clin Gastroenterol Hepatol 5:1447-1453

18. Wilcox WR, Oliveira JP, Hopkin RJ, Ortiz A, Banikazemi M, Feldt-Rasmussen U, Sims K, Waldek S, Pastores GM, Lee P, Eng CM, Marodi L, Stanford KE, Breunig F, Wanner C, Warnock DG, Lemay RM, Germain DP 2008 Females with Fabry disease frequently have major organ involvement: lessons from the Fabry Registry. Mol Genet Metab 93:112-128

19. Deegan P, Baehner AF, Barba Romero MA, Hughes DA, Kampmann C, Beck M; European FOS Investigators 2006 Natural history of Fabry disease in females in the Fabry Outcome Survey. J Med Genet 43:347-352

20. Hogg RJ, Furth S, Lemley KV, Portman R, Schwartz GJ, Coresh J, Balk E, Lau J, Levin A, Kausz AT, Eknoyan G, Levey AS; National Kidney Foundation's Kidney Disease Outcomes Quality Initiative 2003 National Kidney Foundation's Kidney Disease Outcomes Quality Initiative clinical practice guidelines for chronic kidney disease in children and adolescents: evaluation, classification, and stratification. Pediatrics 111:1416-1421

21. Levey AS, Coresh J, Greene T, Stevens LA, Zhang YL, Hendriksen S, Kusek JW, Van Lente F; Chronic Kidney Disease Epidemiology Collaboration 2006 Using standardized serum creatinine values in the modification of diet in renal disease study equation for estimating glomerular filtration rate. Ann Intern Med 145:247254

22. Banikazemi M, Bultas J, Waldek S, Wilcox WR, Whitley CB, McDonald M, Finkel R, Packman S, Bichet DG, Warnock DG, Desnick RJ; Fabry Disease Clinical Trial Study Group 2007 Agalsidase-beta therapy for advanced Fabry disease: a randomized trial. Ann Intern Med 146:77-86 